\title{
Wireless Chemical Sensor Networks for Air Quality Monitoring
}

\author{
Saverio De Vito ${ }^{1}$, Grazia Fattoruso ${ }^{1}$ \\ ${ }^{1}$ ENEA C.R. Portici, Unità UTTP-MDB, P.le E. Fermi, 180055 Portici, Napoli (Italy) \\ saverio.devito@enea.it
}

\begin{abstract}
:
Air quality assessment and monitoring could be efficiently performed by a distributed network of cost effective chemical sensors cooperating for the reconstruction of a chemical image of the sensed environment. The obtainable insights are of paramount importance in many applications ranging from city air pollution monitoring to energy efficiency in smart buildings. However several research studies have highlighted the challenging nature of developing such architecture. Actually, the intrinsic properties of the sensed phenomena, those of the commercially available chemical sensors including power requirement and stability, together with the needed communication infrastructure make a real world implementation of such a system a problem worth of significant investigation efforts. This paper encompass the ENEA-UTTP efforts in providing solutions to several challenges both in indoor and outdoor air quality monitoring setups.
\end{abstract}

Key words: Wireless Sensor Networks, Indoor air quality monitoring, Air pollution Monitoring, Sensor fusion, Energy Efficiency.

\section{Introduction}

The detectable presence of different chemicals in the air, together with their concentration, is a source of valuable information for many applications ranging from pollution monitoring to explosives and drug factories detection. In particular, several gases are considered responsible for respiratory illness in citizens, some of them (e.g. benzene) are known to induce cancers in case of prolonged exposure even at low concentrations [1]. Awkwardly, volatile organic compounds (e.g. formaldehyde) released as off-gas by furnitures adhesives or cleaning agents or by smoking in indoor environments reach concentrations levels that are order of magnitudes higher than in outdoor settings. The estimation of chemicals distribution is hence significantly relevant for citizens safety and consequently for the definition of integrated urban and mobility plans designed to face these problems. However chemicals monitoring can be of paramount importance for security applications e.g. for the detection of explosives and drug factories in cities [2].

However, chemicals monitoring both in outdoor and indoor environment is affected by the peculiarity of chemicals propagation process. To be concise, diffusion and turbulence make a single point of measure ineffective, calling for distributed approaches to chemicals detection and concentration estimation. In many applications this, in turn, require the monitoring task to be fulfilled by a network of wireless (sometime mobile) modules, with the wireless term being related to either connectivity and/or power supply (Wireless Chemical Sensor Networks, WCSN).

Different applications lead to different specific requirements but a number of challenges repeatedly recurs when engineer try to design real world operating WCSN systems. In facts, single module calibration and sensor stability, efficient power usage and cooperative reconstruction seems to be the most common challenges to face both in indoor and outdoor settings.

In this paper we will review these challenges together with the solution proposed by our group during our commitment in the wireless chemical sensing topic.

\section{Module calibration and drift counteraction in outdoor applications}

Nowadays, urban air pollution monitoring is primarily carried out by means of networks of costly fixed stations. These equipments shows significant selectivity, precision and accuracy 
properties but can only be deployed in a rather sparse fashion that hamper the possibility to adequately reconstruct the real distribution of gases and particles concentrations in a complex and turbulent environment such as a city. On the other hand, real time knowledge of the actual pollution concentration pattern in city areas is considered crucial for implementing savvy traffic management and air pollution management policies.

Wireless gas solid state multi-sensor devices can represent a rather interesting tool for densifying the urban pollution monitoring mesh due to their low cost per unit and thanks to their size can be considered suitable for preserving architectural heritage fruition in cities historical centres. The capability to transmit the sensed data wirelessly (e.g. via the ubiquitous $3 G$ infrastructure) make them very appealing since this make their deployment very easy and cost effective.

Unfortunately, the intrinsic selectivity issues, together with the sensibility to environmental condition variationsof solid state sensors can severely limit their precision, accuracy and reliability, in particular when compared with the well established performances of conventional analyzers. Several studies confirms how, when calibrated for single specie quantification in laboratory or mock mixtures, they exhibit poor performances when operating in real conditions [3].

In order to overcome this specific issue, based on the previous literature, we proposed an onfield calibration strategy. The approach was based on the use of a spectrometers based station as a reference for the on-field calibration of a small multisensor device. In our testbed, a feed forward neural network trained using a 10 days measurements set, was capable to gain a relative estimation error for benzene of about $0.01(1 \%)$ during more than 6 months. However the onset of sensor and concept drift effects required a recalibration procedure to be performed every 6 months (see Fig.1). In truth, for this approach to be considered feasible in terms of deployment of hundreds of modules, the possibility to use only a small number of samples as a training set become crucial. An interesting solution could be based on the implementation of on-line learning strategies i.e. having a mobile spectrometer based station provide ground truth values on a regular (or uneven) basis adapting the non- linear calibration of the modules. At the best of our knowledge, this approach has never been implemented, as an extension of this idea, we proposed the use of Semi-Supervised learning process adapting the learnt module calibration exploiting the significant availability of unlabeled samples (see Fig. 2) [4].In a very similar framework, Tsujita et al. proposed an interesting automatic on-field recalibration transfer scheme that could be useful for facing the sensor stability issues [5]. In that paper an easy way to cooperatively share and use the information on base sensor response for continuous calibration was proposed and tested.
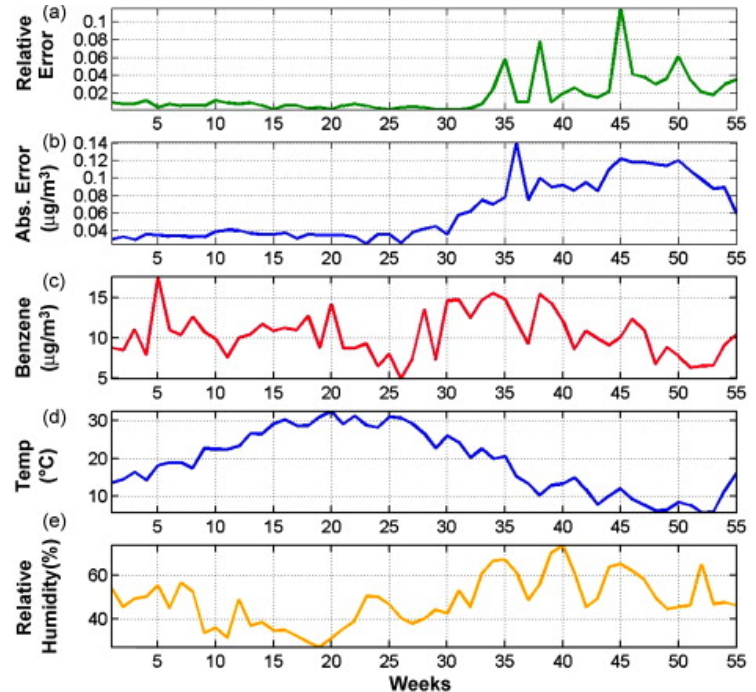

Fig. 1. On Field calibration of a GPRS-enabled MOX based multisensory device for pollution monitoring. Measured error is comparable with the datasheet properties of conventional analyzer for about 6-months after that sensors and concept drift onset severely affected obtained calibration.

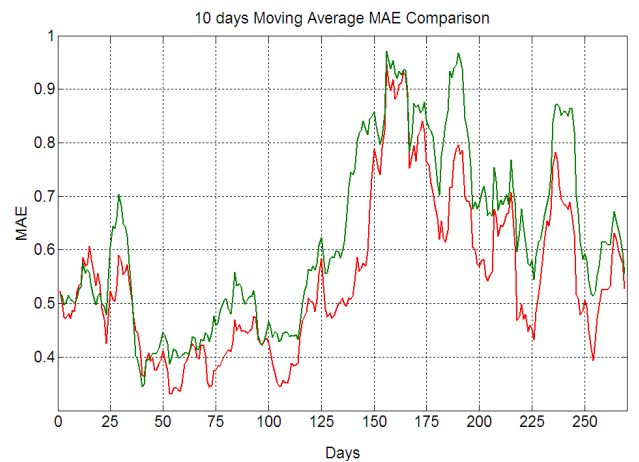

Fig.2. SSL approach to counteract sensor and seasonal concept drifts in an on-field recorded city air pollution database. The CO concentration estimation mean absolute error obtained by a basic NN regressor trained with one day measurements is compared with the results obtained by the SSL based algorithm. The SSL approach achieved a $11.5 \%$ performance gain with respect to the one-year long averaged MAE score. (reprinted from [4]).

\section{Efficient power usage in indoor air quality monitoring applications.}

In the last decade, several research papers investigated the possibility to monitor the indoor 
air quality for multiple applications scenario ranging from safety to security and energy efficiency. Just as an example, e-nose has been tested on the ISS in search for possible precursors of dangerous on-board fires originating from short circuits and cable degradations. Furthermore HVAC systems could be efficiently controlled and operated by constantly monitoring Air quality and Comfort. Again, due to the complexities of chemical signal propagation in indoor environments, the use of a distributed network of analyzers is mandatory. The possibility of wireless nodes to be deployed wherever possible is also crucial for such scenarios. However, one of the main challenge to be faced in this scenario is module survivability in terms of energy supply when dealing with true wireless systems, operated on batteries. Often the primary source of energy consumption is the chemical sensors itself. Some MOX sensors, for example, consume up to $400 \mathrm{~mW}$ on their active stage. These figures prevents their use on battery operated modules unless some different operating strategy is devised. On their 2009 paper, S. Bicelli et al. described a low power operating strategy of a commercial MOX sensor that lead the sensor to drain an optimal energy level without destroying accuracy properties [6].

The second source of energy drain in wireless chemical sensor modules is certainly the wireless communication facility. Transmitting data is, in fact, very costly in terms of energy, transmitting non-relevant information can be especially nasty when dealing with mesh topologies. Actually the use of mesh topologies together with adaptive routing is extremely important for ensuring extended coverage and robustness to module failures. However, in this architecture, a data packet travels along multiple nodes before reaching is final destination on base station. Unuseful packets, hence, when routed throughout the network, drains batteries of all involved nodes. Sensor censoring, that is the capability to discharge non relevant acquisition, is a possible solution to the cost of non relevant data transmission. However simple threshold based approaches may fail or be difficult to tune in the chemical sensor scenario due to the complex response of the sensors array itself. One of the possible solution is to provide the node with local intelligence in order to establish the relevance of the sensed data in that precise time instant and position. In this sense we proposed to embed a local computational intelligence software component actually implementing an on board neural network to make the module capable to locally estimate the concentration of two mock pollutants (Acetic Acid and Ethanol) for which its sensor array was calibrated [7].

For example the module could be trained to transmit data when relevant concentrations of one of the two pollutants ignoring high concentration of the other one simulating the presence of a dangerous pollutant. In this case a badly tuned sensor response/threshold based approach would have generated a significant number of false positive.

\section{Chemical Sensor fusion: 3D chemical image reconstruction}

The capability to reconstruct the concentration of chemicals in a $3 D$ environment is the final stage in an integrated indoor or outdoor chemical monitoring system. This capability may allow, for example, to locate possible gas spills in safety applications, to locate highly polluted areas in outdoor environment, to divert car traffic for air pollution mitigation, etc.

Authors like Diamond et al. have tackled the wireless chemical sensing scenario demonstrating the capability of detection of single sensor platforms in single chemical detection tests while Liliental et al. pursued a multi sensor robot approach addressing particularly the 3D source declaration problem [8-9].In a recent paper, we proposed to investigate the use of a mesh of intelligent wireless e-noses for 3D quantitative indoor air quality reconstruction in presence of a gas mixture [10].In that study we extended our work pursuing the $3 \mathrm{D}$ gas concentration mapping of acetic acid - ethanol mixtures (as VOC pollutant simulants) in ambient air by a mesh of wireless electronic noses coupled with the previously mentioned sensor fusion algorithm. $3 \mathrm{D}$ reconstruction was obtained by adapting the Lilienthal approach, originally designed to deal with a single pollutant and a single moving robot, to the use of a number of fixed measurement points. In fact this approach will guarantee a timely response wrt to the single mobile robot approach that need the robot to move scanning the environment. On the other hand, the possibility to move will guarantee a better coverage of the sensed environment.

A set of 4 w-noses relying on 4 MOX sensors array was developed using Figaro 2602, 2600, $2 \times 2620$ sensors. The sensors array have been assembled on a signal conditioning board and connected to a commercially available WSN platform (Crossbow TelosB mote) and then integrated in a compact plastic case for free gas flow operation. All the platforms were calibrated in lab conditions using their response to artificial Acetic Acid/ Ethanol mixtures synthetized in a climatic chamber and a NN based on-board 
regressor. Each module was then found capable to estimate the local concentration of the analytes with an error ranging from $1 \mathrm{ppm}$ (Acetic Acid) to 10ppm (Ethanol). Then they were deployed in an ad-hoc measurement chamber and exposed to different concentration of the two analytes. The actual estimation of each nodes were fused together by a 3D Gaussian estimation algorithm to reconstruct a real time 3D picture of the gas concentrations (see Fig. 3). An unsupervised collaborative parameters tuning was devised to adapt the algorithm parameters during the operative life of the deployment.
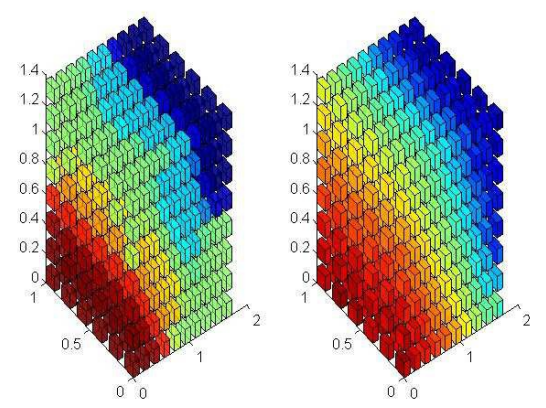

Figure 3: Real-time 3D ethanol (right) and acetic acid (left) concentration reconstruction (computed @ datasink) using a 4 w-nose deployment in the glass box experimental setup.

\section{Conclusions}

In this paper we have shortly reviewed three main challenges towards the design of a low cost, pervasive, wireless, chemical sensing network capable to operate in real world environments for different applications. Calibration, sensors and concept drifts issues have been discussed for outdoor scenarios while energy efficiency and 3D reconstruction have been analyzed for indoor settings. Possible solutions, investigated by our and other groups have been reviewed and compared.

\section{References}

[1] D. Dockery, C.A. Pope, X. Xu, F. Speizer, J. Schwartz, An associationbetween air pollution and mortality in six US cities, N. Engl. J. Med. 329

[2] Project-FP7-Lotus http://www.ait.gr/export/sites/default/ait_web_site/ pdf_projects/LOTUS.pdf

[3] M. Kamionka, P. Breuil, C. Pijolat, Calibration of a multivariate gas sensingdevice for atmospheric pollution measurement, Sens. Actuators B Chem. 18(2006) 323-327.

[4] S. De Vito et al. Semi-Supervised Learning Techniques in Artificial Olfaction: A Novel Approach to Classification Problems and Drift Counteraction, Accepted for publication in IEEE Sensors.

[5] W. Tsujita, A. Yoshino, H. Ishida, T. Moriizumi, Gas sensor network forair-pollution monitoring, Sens. Actuators B: Chem. 110 (2005) 304-311.

[6] S. Bicelli et al. "Model and Experimental Characterization of the Dynamic Behavior of LowPower Carbon Monoxide MOX Sensors Operated With Pulsed Temperature Profiles", IEEE Trans. Instrumentation and Measurement, May, 2009, Vol. 58, N. 5, pp. 1324-1332, ISSN 0018-9456, DOI 10.1109/TIM.2009.2012940.

[7] S. De Vito et al., Wireless Sensor Networks for Distributed Chemical Sensing: Addressing Power Consumption Limits with On-Board Intelligence. IEEE Sensors Journal, Vol. 11, Issue 4, pp.947955

[8] D. Diamond,S. Coyle, S. Scampagnani, J. Hayes, Wireless Sensor Networks and Chemo/Biosensing, Chem. Rev., 108 (2), pp 652-679 (2008).

[9] Achim J. Lilienthal, Amy Loutfi and Tom Duckett, Airborne Chemical Sensing with Mobile Robots. Sensors, 6, pp. 1616-1678 (2006).

[10] S. De Vito et al., Procedia Engineering, Volume 25, 2011, Pages 84-87, ISSN 1877-7058, 10.1016/j.proeng.2011.12.021. 